DOI: https://doi.org/10.46296/yc.v5i8edespen.0103

\title{
INNOVACIÓN ESTRATÉGICA PARA EMPRESAS DE OCÉANO ROJO. ALTERNATIVAS PARA UNA TRANSICIÓN EXITOSA.
}

\section{STRATEGIC INNOVATION FOR RED OCEAN COMPANIES. ALTERNATIVES FOR A SUCCESSFUL TRANSITION.}

\author{
Avellán-Moreira Jeniffer Stephania ${ }^{1 *}$; Vegas-Meléndez Hilarión José ${ }^{2}$ \\ ${ }^{1}$ Ingeniera en Administración de Empresas, Pontificia Universidad Católica del \\ Ecuador, Sede Manabí. Portoviejo, Ecuador. Correo: javellan8550@pucesm.edu.ec \\ ORCID ID: https://orcid.org/0000-0001-7889-5818 \\ ${ }^{2}$ Docente e investigador de pre y posgrado de la Pontificia Universidad Católica del \\ Ecuador, sede Manabí. Portoviejo - Ecuador. Correo: hvegas@pucesm.edu.ec. \\ ORCID ID: https://orcid.org/000-0002-8526-2979
}

\begin{abstract}
Resumen
El objetivo principal de la presente investigación es contextualizar el proceso de innovación estratégica en empresas de océano rojo como alternativa de diferenciación, garantizándole de esta manera una ventaja sostenible ante su competencia. La perspectiva es de carácter inductivo, bajo un enfoque de tipo cualitativo, con un nivel descriptivo documental. El instrumento de recolección de información responde a la aplicación de una entrevista a profundidad al Presidente de la Cámara de Comercio de la ciudad de Portoviejo, capital de la provincia de Manabí (Ecuador) como actor relevante, así como investigadores conocedores de la temática a través de investigaciones previas y acciones empresariales (consultorías), entendidos en esta investigación de igual forma como actores relevantes. Con el fin de verificar, comprender e interpretar el planteamiento de los entrevistados se utilizó el análisis hermenéutico que permitió la captación plena del discurso de los actores en los diferentes contextos: Ecuador, México y Venezuela. La investigación concluye con la importancia de incorporar los avances tecnológicos como estrategias de diferenciación que le permite a las empresas ganar la mayor cuota del mercado con productos o servicios únicos y difíciles de imitar, revelando de esta manera la necesidad de abrir espacios al capital intelectual como eje estratégico de toda organización anclada en los denominados océanos rojos o mercados saturados, garantizando así cada uno de los procesos de desarrollo e innovación.
\end{abstract}

Palabras clave: Innovación, estrategia, océano rojo, factor diferencial, Teoría Fundamentada.

\begin{abstract}
The main objective of this research is to contextualize the strategic innovation process in Red Ocean companies as an alternative for differentiation, thus guaranteeing it a sustainable advantage over its competition. The perspective is inductive in nature, under a qualitative approach, with a descriptive documentary level. The information collection instrument responds to the application of an in-depth interview with the President of the Chamber of Commerce of the city of Portoviejo, capital of the province of Manabí (Ecuador) as a relevant actor, as well as researchers with knowledge of the subject through of previous research and business actions (consultancies), understood in this research in the same way as relevant actors. In order to verify, understand and interpret the approach of the interviewees, the hermeneutical analysis was used that allowed the full capture of the discourse of the actors in the different contexts: Ecuador, Mexico and Venezuela. The research concludes with the importance of incorporating technological advances as differentiation strategies that allow companies to gain the largest market share with unique and difficult-to-imitate products or services, thus revealing the need to open spaces for intellectual capital such as strategic axis of any organization anchored in the so-
\end{abstract}

Información del manuscrito:

Fecha de recepción: 16 de noviembre de 2020.

Fecha de aceptación: 08 de enero de 2021.

Fecha de publicación: 29 de enero de 2021. 
called red oceans or saturated markets, thus guaranteeing each of the development and innovation processes.

Keywords: Innovation, strategy, red ocean, differential factor, Grounded Theory.

\section{Introducción}

"iSocorro! Mi océano se está volviendo rojo, es una frase que capta a la perfección el sentimiento del que se hacen eco tan frecuentemente los ejecutivos de las empresas de todo el mundo" (W Chan \& Mauborgue, 2015), haciendo referencia a esta frase, a medida que aparecen más competidores, las oportunidades de crecimiento son mínimas, los productos se tornan homogéneos y se produce una competencia feroz que colorea y convierte el agua en un océano rojo. "Los océanos rojos representan los mercados saturados, donde las oportunidades de crecimiento son poco frecuentes, puesto que en ellos intervienen muchos actores que combaten ferozmente para ampliar su cuota de mercado" (W Chan \& Mauborgue, 2015), importante descripción por parte de estos autores, donde los paradigmas y las reglas del juego son conocidas y aceptadas; sin embargo, y en contraste, Borghino (2012) indica que "La nueva regla del juego en mercados saturados es que ya no hay regla" (pág. 14); esto deja entender que los mercados de océano rojo o saturados son tan complejos que no hay una definición clara en su comportamiento. Existe una gran cantidad de satisfactores, múltiples alternativas que cubren más de una necesidad, donde se tiene la oportunidad de adquirir productos rápidamente, el cual, al existir más de una opción de compra, los productos comienzan a ser más efímeros. Así, las empresas deben transformar las amenazas que se puedan presentar en oportunidades que les permita sobrevivir al mercado saturado en el que se encuentran o mediante alternativas iniciar la transición hacia un océano azul con más oportunidades. Ejemplo de esto tenemos la visión estratégica de la Cámara de Comercio de la Ciudad de Portoviejo (provincia de Manabí), como actor importante de la investigación, en la 
que muchas de sus empresas o comercios se encuentran ancladas en el inmenso océano rojo, reconocidas por su funcionamiento, presencia y sostenibilidad en el mercado, en muchas de ellas se ha observado una fuerte ascendencia en el manejo de estrategias basadas en precios, cuyo principal objetivo es ganarles a sus competidores en base a este criterio.

El océano rojo, siendo un mercado que nace de la definición del océano azul, el cual es considerado como un mercado sin explorar, siendo el océano rojo su contraparte; se compone por aquellas industrias que mantienen saturado el mercado y que basan su accionar en enfrentar a la competencia bajo diversas estrategias de marketing donde buscan ganar más clientes 0 , en su defecto, quitarles clientes a sus competidores, distinguiéndose por ser desleal frente a las marcas por motivos de precios o facilidades que se establezcan por parte de la oferta en productos o servicios.

En virtud de la situación de la lucha por la guerra de precios, muchas empresas ancladas en este océano han tenido un éxito muy esquivo, llevándolas a la desaparición por caer en las trampas del mismo. Ahora bien, no todas las empresas tienen la capacidad de innovar, aplicar nuevos conocimientos, de lograr una ventaja competitiva por la ausencia de su principal ingrediente, la innovación estratégica. De esta manera, como hace mención Oppenheimer (2014) "la innovación se está democratizando, y cada vez está más cerca de nuestro alcance". La innovación no se basa solo en innovar y obtener aquel factor diferencial, sino en la capacidad que poseen las empresas de que aquella innovación afecte e impacte de una manera estratégica en el mercado y el entorno en el que opera, generando altos niveles de productividad; así, la innovación adquiere un valor estratégico, transcendiendo las barreras, situándose en todos los niveles de competitividad, siendo éste el papel más importante dentro de la concepción de cualquier empresa, convirtiéndose en el motor de transformación y crecimiento de quienes quieren permanecer en mercados saturados. 
Esta innovación va de la mano de la tecnología, donde interviene el conocimiento, logrando construir un nuevo camino para llegar a la meta planteada, siendo una necesidad que, a través de proponer nuevas ideas, permitirá avanzar, hacer algo diferente a lo actual y que el futuro pueda ser mejor que el presente, que es lo que se busca lograr en el océano rojo. Entonces, "la innovación estratégica se considera cada vez más un proceso complejo y dinámico que comprende muchos factores sociales y económicos, y un gran número de personas, empresas e instituciones". (Pulgarín \& Pineda, 2011)

La innovación estratégica generalmente permitirá a las empresas expandir sus horizontes, hacer cambios, contribuir a la realización de los objetivos propuestos, responder a las oportunidades potenciales del mercado y ser capaces de liderarse con características que superen a los competidores, creando así valor. Por lo tanto, la innovación estratégica establecerá un umbral de diferencia sostenible en el tiempo porque es lo suficientemente amplio como para asegurar la competitividad y sostenibilidad del mercado.

Interesante análisis digno de mencionar por Borghino (2012) "los competidores están al acecho de un momento de incertidumbre, un instante de distracción, un error de estrategia para asestar su golpe". (pág. 24), el autor hace mención que los competidores están al acecho de un momento de incertidumbre, de un error, por eso es importante que analicen el riesgo o problemas que enfrenta la empresa, los cuales serán determinantes para asegurar su permanencia en el mercado, por lo que ignorarlo es más perjudicial que la aparición de nuevos productos o competidores.

El desafío actual de toda empresa anclada en el océano rojo es reinventarse, lograr esa conexión entre los propósitos $\mathrm{u}$ objetivos planteados desde sus inicios, analizando de manera exhaustiva las últimas tendencias que surgen en el mercado en el que operan, conociendo de ésta manera a qué se enfrentan y los riesgos que deben afrontar, compitiendo de forma estratégica, no solo en base a precios, sino en tecnología, 
innovación y en aquel factor diferencial, ganando la mayor parte del mercado, ya que, en el escenario actual, los consumidores son cada vez más exigentes. Otro de los problemas que surge en estas empresas, es la imitación constante, productos genéricos que no crean el nivel óptimo de diferenciación, logrando la fidelización inmediata del cliente (incluso en un tiempo en el que existe un gran número de satisfactores). En esta última situación, la investigación procura contextualizar el proceso de innovación estratégica en empresas de océano rojo como alternativa de diferenciación, que le permita a las mismas, ampliar sus conocimientos sobre las características más relevantes de la competencia, analizando los procesos de planeación e implementación de diferentes estrategias innovadoras aplicadas en éstas empresas que inciden en la creación de valor para los participantes tanto internos como externos, siendo determinantes en el crecimiento económico y competitivo de las empresas ancladas en este océano, logrando una ventaja sostenible, generando cambios con el objetivo de adaptarlos a nuevos entornos donde existe una alta competitividad.

\section{Innovación para la diferenciación estratégica}

Cabe mencionar, que la innovación, siendo fundamental para el éxito de cualquier empresa, cumple una función clave en la definición de las estrategias basadas en la diferenciación. Es necesario partir de uno de los principales e importantes precursores del tema, Porter (2015) indica que "los dos tipos básicos, combinados con el ámbito de las actividades que las empresas intentan obtenerlos, dan origen a tres estrategias genéricas para lograr un desempeño superior promedio de la industria: 1) liderazgo en costos, 2) diferenciación en los costos y 3) enfoque en la diferenciación". Las estrategias genéricas hacen referencia en cómo obtener una ventaja competitiva, planteando para ello rutas alternativas que le permitan a la empresa alcanzar un desempeño superior a su competencia, que, en este caso, va a depender de la estructura del sector industrial en el que operan. Es importante mencionar que las empresas que aplican las estrategias 
genéricas, al ser puras y fáciles de imitar, deben cuidar las ventajas con las que cuentan, a fin de cuidar que sus competidores directos las imitan con facilidad.

Cada una de las estrategias genéricas planteadas por Porter (2015), son importantes para el desempeño de la empresa que opte por aplicarlas, pero para el desarrollo de la investigación, se estudiará la vertiente basada en estrategias de diferenciación. En las estrategias de diferenciación, Porter (2015) menciona que la empresa "intenta distinguirse dentro de su sector industrial en aspectos bastante apreciados por los compradores. Escoge uno o más atributos que juzgue importantes y adopta un posicionamiento especial para atender esas necesidades. Ve premiada su singularidad con su mayor precio" (p.41).

La diferenciación, como lo menciona Gómez (2017) "es distinguir un producto o servicio del resto de sus competidores, buscando hacerlo más atractivo a los ojos de su mercado objetivo, para que lo prefieran" (pág. 99). Partiendo de esta definición, la lógica de la estrategia de diferenciación es que los productos, acompañados con el servicio que ofrecen al comprador, lo perciban como único, que los atributos que proyectan sean superiores en el sector que operan, centrándose en satisfacer cada una de esas necesidades con el objetivo de obtener una mayor demanda y aumentar los precios en el caso de que los consumidores reconozcan que sus productos sean diferenciadores.

Es evidente que de la innovación nace la necesidad de diferenciarse, la evolución de los mercados y las exigencias de los consumidores invita a las empresas a involucrar el ingrediente clave, la innovación basada en la diferenciación en cada uno de sus procesos y actividades, el cual, al incluirla, se puede crear valor, lealtad hacia la marca, hacer de la competencia algo irrelevante pero no menos importante. En este caso, Gómez (2017), sugiere evitar competir por precio "aparte el producto o servicio de los demás, poniéndolo en otra categoría. No es comparable. El valor que representa para el cliente supera su relación costo - beneficio por encima de la competencia" (pág. 100). Muchas empresas, en este caso, las 
ancladas en el océano rojo, basan sus estrategias en precios, lo que puede convertirlas en irrelevantes para los clientes, pero aplicar estrategias basadas en la diferenciación, requiere en muchos casos, ser radical para que los consumidores o sus competidores logren notarlo, rompiendo de esta manera los estereotipos de creer que mantenerse en lo mismo es más seguro y no el salir de su zona de confort, siendo lo contrario, cuando una empresa tiene claro el factor diferencial, existe claridad y seguridad, y se vuelve poderosa, comunicándolo al mercado de una manera efectiva y significativa para el mercado. El factor diferencial se va perfeccionando con el pasar del tiempo, nunca es definitiva, va surgiendo en base a los cambios que se presenten en el mercado que opera y está inmersa en los pequeños detalles, pero muy bien ejecutados, logrando fidelizar a los clientes.

W. Chan \& Mauborgne (2018) hacen mención que "los océanos rojos son todos los sectores que existen hoy y en los que luchan la mayoría de las empresas", para los autores, esta teoría sobre la competencia en el mercado entre empresas ancladas en el mismo, la experiencia, el ser creativas, no basta. De esta manera, la innovación para la diferenciación estratégica, pueden marcar un antes y un después dentro de las empresas que las aplican, adaptando para ello una perspectiva de océano azul, expandiendo sus horizontes, mediante la comprensión de qué necesita el consumidor y cómo satisfacer las mismas. Con este campo de visión ampliado, una nueva frontera de valor para el producto, servicio y cliente se asegura el camino en la dirección correcta. Muchas de las empresas ancladas en mercados saturados $u$ océanos rojos, el trabajo estratégico que desempeñan, lo basan en función de los precios, más no en la diferenciación.

La innovación juega un papel importante en el proceso de la diferenciación estratégica, pero solo cuando las características diferenciadoras aplicadas al producto son difíciles de imitar por la competencia, el cual va a permitirles ser superiores en términos de preferencias, a través de productos adaptados preferiblemente a las demandas del mercado. El reto para 
cualquier empresa anclada en el océano rojo es saber comunicar el factor diferencial, el usar mensajes genéricos, le llevan a que los clientes o consumidores terminen decidiéndose por el precio, más no por el factor diferencial que pretenden mostrar al mercado, siendo ante la vista del mismo, un producto más. Así, "en los mercados actuales existen segmentos con altas exigencias de servicio que se constituyen en una oportunidad" (Chirinos Cuadros \& Rosado Samaniego, 2016), esta oportunidad se ve reflejada en el factor diferencial, donde los consumidores buscan la verdad, empresas y marcas que sean honestas en base a lo que proyectan, que les brinden una historia y experiencias únicas, de esta manera, son más propensas a pagar por algo que le genere satisfacción y gratificación al adquirirlo, tal como lo menciona Gómez (2017) al considerar que "los diferenciales son transitorios. La estrategia necesita evolución continuamente, porque los clientes y los mercados están cambiando más rápido que nunca”. (pág. 192). En base a la estrategia, Castro Monge (2010) hace mención que "se introducen las apropiadas características materiales como la estructura, los sistemas 0 los procesos", configuración de los recursos que posee la empresa en base a los cambios del entorno, que van a permitir alcanzar el éxito deseado en la aplicación de la misma.

\section{Disertación de alternativas para una bitácora exitosa}

Las empresas ancladas en océanos rojos sin duda requieren de estrategias que bien le permitan no solo mantenerse como dominantes de este mercado, sino que a su vez es imprescindible asumir estrategias que les genere alternativas para un tránsito más asertivo hacia un mercado de océano azul, que en definitiva es el objetivo de toda empresa que se encuentra en el océano rojo y que estratégicamente basa sus acciones en la innovación. A partir de este espacio de discusión, luego de un proceso de saturación teórica, se resaltan diversas posturas por parte de los investigadores que intentan abrir una zona de disertación para nuevas alternativas dentro del contexto de un mercado rojo. 
Se considera que el principal reto de una empresa exitosa radica en la capacidad de adaptación a los cambios y dar respuesta ante los desafíos empresariales que se presentan, utilizando y sacando ventaja de la información que se genera dentro y fuera de la organización, canalizando los recursos que tiene a su favor, convirtiéndolas en oportunidades mediante la automatización de tareas de mayor relevancia en conjunto con los miembros de la empresa.

Por otro lado, la innovación va muy ligada a preocuparse por averiguar las necesidades del cliente final $y$ buscar la mejor forma de satisfacerlas. Se considera que una empresa al no innovar estratégicamente corre el riesgo de que sus productos se tornen homogéneos. La innovación estratégica afecta directamente a la capacidad de liderar en el mercado con características que superen a los competidores, de no implementarla en la empresa, no asegurará su permanencia en el mercado por la aparición de nuevos competidores que lideran el mismo con productos diferenciadores, con valor agregado.
Desde este espacio de discusión, se hace necesario resaltar la importancia de conocer el entorno (oportunidades y amenazas), teniendo una visión amplia del mercado. Dentro de este punto es vital conocer a los competidores o proveedores realizando alianzas para bajar los costos. Es necesario optar por una estrategia comercial, que permitirá lograr la diferenciación y enfocarla en un segmento determinado, resaltando el aspecto o característica del producto o servicio que se ofrece. Además, se requiere implementar una estrategia corporativa, la cual conlleve conectar a la empresa con su entorno, aplicando estrategias de especialización en el producto 0 servicio actual que se ofrece dentro del mercado en el cual se actúa, o las estrategias de desarrollo de productos, ya sea mejorar uno existente o lanzar al mercado nuevo, distintos a los productos o servicios actuales de la empresa.

En definitiva, es imperativo explorar más allá de la demanda existente fijándose en los que no son clientes y en todas las características comunes de los clientes actuales; fortalecer la gestión y cimentar 
nuevas iniciativas comerciales; ingresar a nuevos sectores distintos al que se desenvuelve; crear productos o servicios alternativos que también resulten atractivos al consumidor o usuario; así como, y no menos importante, promover experiencias con grandes conexiones emocionales entre la marca y el cliente, logrando fidelizar.

\section{Constructo metodológico para un océano rojo}

La investigación se desarrolló bajo un enfoque de tipo cualitativo, acorde con lo propuesto por los autores Muñoz Rocha (2015) "La investigación cualitativa se puede identificar como una investigación donde la recolección de datos no demanda su medición numérica. La actividad indagatoria se centra en los hechos y su interpretación", dado que permitirá describir de manera objetiva los acontecimientos, experiencias, en las que se encuentre incurso, apartando todo tipo de creencias, religión y postura que pueda desviar el curso de la investigación; la investigación asume un compromiso de utilizar las técnicas correspondientes a garantizar esta perspectiva metodológica buscando un análisis y una interpretación critica durante todo el proceso.

El alcance de la investigación es descriptivo, ya que "pretende dar cuenta de las características de un fenómeno $u$ objeto sujeto a investigación, de sus propiedades, características atributos, componentes, elementos y relaciones entre ellos" (Muñoz Rocha, 2015), orientada a develar los principales componentes, características y atributos que tiene un océano azul, contrastando con el estudio que adelanta la investigación relacionada con el océano rojo, dándose a conocer a su vez, todos estos elementos de dicho océano, describiendo las características, propiedades y detalles relacionadas al mismo.

El diseño de esta investigación se basa en una revisión documental de forma sistemática, permitiendo recopilar teorías, postulados y discusiones académicas, las cuales sustentan la idea investigativa en términos argumentativos, dando viabilidad a la concepción de un océano rojo sobre la innovación estratégica para empresas ancladas 
en estos océanos. La población documental

la conforman referencias bibliográficas, básicamente de artículos científicos especializados nacionales e internacionales, como lo sustenta Muñoz Rocha (2015) "el investigador se basa en fuentes bibliográficas o en general documentales, por la imposibilidad de obtener información de forma directa o de primera mano".

Respecto a las técnicas de investigación aplicadas en esta investigación, se tiene la entrevista, siendo una "conversación dirigida al logro de determinados objetivos, que se puede establecer con una persona (individual) o grupo de personas (grupal)" (Hernández Escobar, y otros, 2018, pág. 123), permitiendo obtener respuestas a las interrogantes planteadas mediante un diálogo con expertos y actores relevantes para la investigación, obteniendo información de parte del mismo, respecto a las estrategias implementadas para ganar clientes de la competencia o en tales casos, quitar los clientes de la misma y mantenerse en los mercados saturados.
La entrevista busca:

Obtener las concepciones personales de los entrevistados sobre la situación objeto de la investigación en medio de un esfuerzo de inmersión $y$ reinversión con intimidad $y$ familiaridad para sinceramente reconstruir a través de la discursividad el punto de vista (perspectiva) del informante (o actor relevante) en el cual se ubica la investigación y su contexto (Vegas, 2016, pág. 79)

Se consideró para efectos de las entrevistas al principal ejecutivo de la Cámara de Comercio de la Ciudad de Portoviejo (Provincia de Manabí), como lo es su Presidente el Economista Diocles Valeriano, aplicando una entrevista en profundidad basada en un cuestionario en la que se presentan 4 interrogantes, obteniendo las respectivas respuestas por parte del entrevistado, acá se identifica el discurso de este importante actor social, se presenta un ejercicio de comprensión e interpretación del planteamiento expresado por el entrevistado a fin de generar 
resultados y conocimientos para la investigación.

Además, se contó con la participación de académicos e investigadores conocedores del tema, donde se revelan sus experiencias sobre estrategias de innovación para mercados saturados u océanos rojos, permitiendo visualizar elementos conectores para poder llegar a las posibles resultados y conclusiones.

Es importante señalar que los criterios de selección de los entrevistados, en el caso de estudio, son los presidentes encargados en virtud de las responsabilidades y experiencias que puedan mostrar en el ejercicio de sus funciones estratégicas y tácticas; por otro lado, los criterios utilizados para la selección (escogencia) de los académicos-expertos está basada en las investigaciones desarrolladas en el campo de la gerencia, su grado académico (PhD), y su experiencia en el campo de las asesorías y consultorías, siendo estos dos últimos la Dra. Diana Ortiz (México) y el Dr. Víctor Hugo Pinto (Venezuela), quienes destacan en el ámbito académico y científico en el aporte de los mercados saturados (océanos rojos), además de una visión distinta que puede contrastar con las posturas de los principales teóricos en la que se sustenta la investigación y del actor entrevistado representante de la Cámara de Comercio mencionada

anteriormente.

La investigación establece una ordenación conceptual desde la perspectiva de los informantes, para ello se estableció un recorrido que permitiera la configuración de las categorías resultantes, dando lugar a los descriptores gerenciales que sustentan las prácticas estratégicas que facilitan la innovación y la diferenciación en los mercados rojos, todo ello basado en lo expuesto por Gadamer (1998) en la necesidad de comprensión, interpretación y aplicación de lo manifestado por estos mismos informantes en términos hermenéuticos.

La tabla 1 establece, de manera sucinta, las resultantes categorías producto del análisis desarrollado por los investigadores, observándose los códigos in vivo según lo expresado por cada entrevistado, así como los 
descriptores que se generan al final de la triangulación de los comentarios entre referentes teóricos, actores y expertos académicos.

Tabla 1. Categorías y Descriptores Emergentes de la Entrevista en Profundidad.

\begin{tabular}{|c|c|c|c|}
\hline Entrevistado & Códigos en vivo & Categorías & Descriptores \\
\hline $\begin{array}{l}\text { AC: Diocles } \\
\text { Valeriano (2020) }\end{array}$ & $\begin{array}{l}\text {-Compensación } \\
\text {-Cambios } \\
\text { tecnológicos } \\
\text {-Centros } \\
\text { comerciales } \\
\text { capitalizan el } \\
\text { mercado } \\
\text {-Alternativas no } \\
\text { optativas } \\
\text {-Productos } \\
\text { primarios sin valor } \\
\text { agregado } \\
\text {-Descomposición } \\
\text { empresarial } \\
\text {-Asociación con } \\
\text { mercados } \\
\text { internacionales } \\
\text {-Calidad y precio }\end{array}$ & $\begin{array}{c}\text {-Cambios en el } \\
\text { mercado } \\
\text {-Comercio } \\
\text { tecnológico } \\
\text {-Riesgos no } \\
\text { visionados } \\
\text {-Alianzas } \\
\text { estratégicas } \\
\text {-Marca }\end{array}$ & $\begin{array}{c}\text { - Visibilizar la } \\
\text { empresa frente al } \\
\text { entorno } \\
\text {-Gestión } \\
\text { empresarial basada } \\
\text { en el } \\
\text { emprendimiento y } \\
\text { el cambio } \\
\text { tecnológico }\end{array}$ \\
\hline $\begin{array}{l}\text { EAI: Diana Ortiz } \\
\text { Torres }(2020)\end{array}$ & $\begin{array}{l}\text {-Oferta y demanda } \\
\text {-Valor agregado } \\
\text {-Facilitar contacto } \\
\text { con clientes } \\
\text {-Disminuir tiempos } \\
\text { de entrega } \\
\text {-Sobrevivir a la } \\
\text { competencia } \\
\text {-Compromiso por } \\
\text { parte de TTHH } \\
\text {-Servicio optimizado } \\
\text {-Competencia } \\
\text { desleal } \\
\text {-Impacto real en el } \\
\text { medio ambiente }\end{array}$ & $\begin{array}{l}\text { - Digitalizar } \\
\text { procesos } \\
\text {-Capital humano e } \\
\text { intelectual } \\
\text {-Espíritu de } \\
\text { servicio } \\
\text {-Filosofía } \\
\text { empresarial } \\
\text {-Ambientalmente } \\
\text { responsables } \\
\text { - Desarrollo } \\
\text { sustentable }\end{array}$ & $\begin{array}{l}\text { - Empresas } \\
\text { comprometidas con } \\
\text { el ser humano y el } \\
\text { medio ambiente }\end{array}$ \\
\hline
\end{tabular}




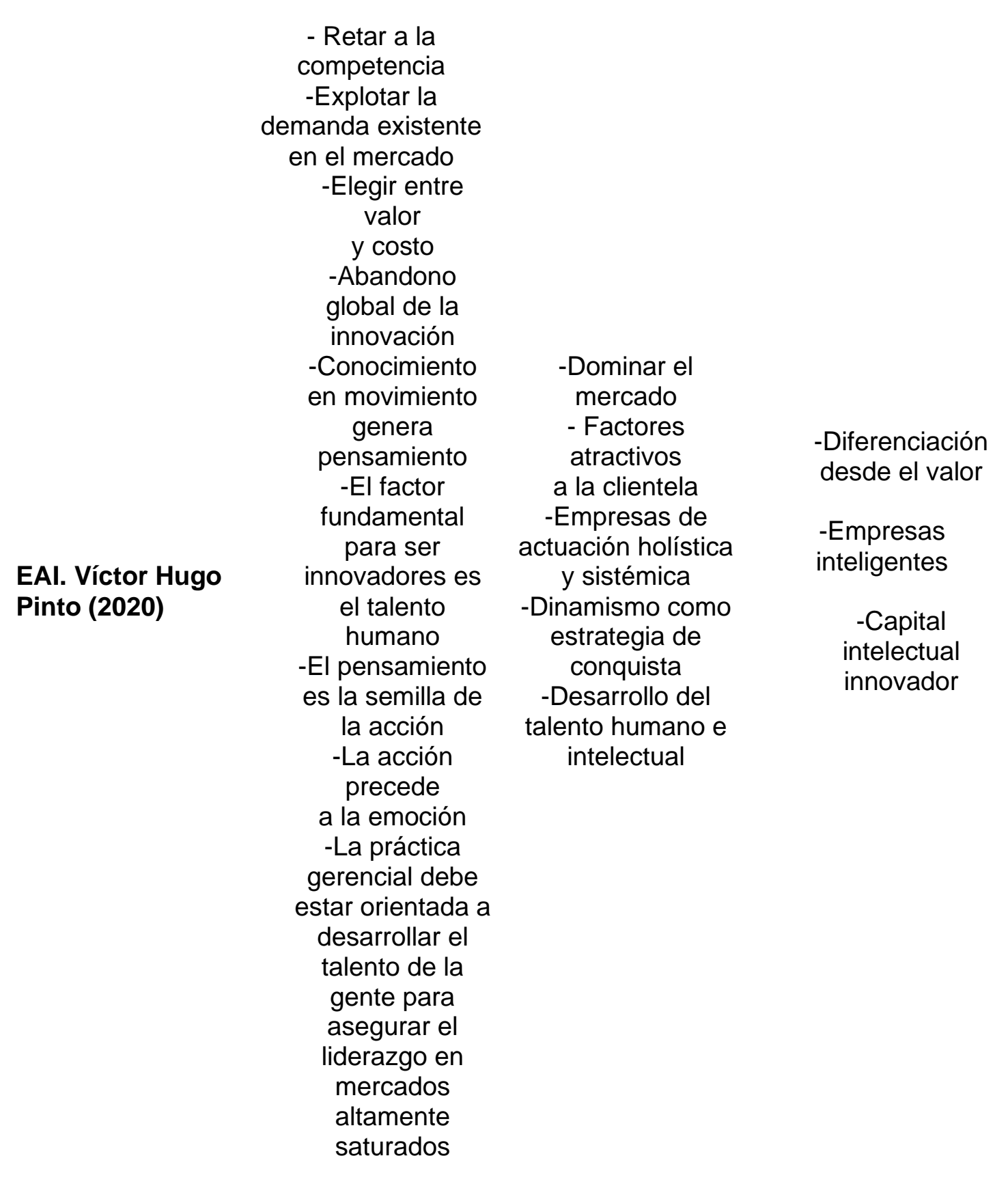

Fuente: Avellán \& Vegas (2020) a partir de entrevistas en profundidad.

Notas: AC: Actor de cambio; EAl: Experto Académico Investigador.

Luego de desarrollar el proceso de categorización en la que resultan los descriptores resultantes (tabla 1), los investigadores proceden a presentar los resultados de la investigación luego de la triangulación correspondiente entre teorías relacionadas, posturas de los entrevistados y análisis hermenéutico- al describir cada componente como indicativo del camino a seguir por quienes tienen la 
oportunidad de gerenciar situaciones similares, buscando alternativas para un mejor desenvolvimiento de sus empresas en los llamados mercados saturados (océanos rojos).

La investigación genera los siguientes descriptores estratégicos:

- Visibilizar la empresa frente al entorno.

El entorno es un condicionante importante para cada empresa, su visibilidad debe ser una prioridad estratégica en la que esta debe sentirse y hacerse parte de la ciudadanía global; la empresa tiene un rol importante como uno más de la sociedad, y es el de contribuir con su desarrollo.

- Gestión empresarial basada en el emprendimiento y el cambio tecnológico.

La empresa no solo debe gestionar su funcionamiento, es necesario pensar siempre como emprendedor, aún después de estar funcionando desde hace años en el mercado. La mentalidad de emprendedor constante permite a empresas de océano rojo enfrentar las adversidades con sentido de innovación y reconocimiento de la tecnología como principales aliados para su sostenibilidad.

- Empresas comprometidas con el ser humano y el medio ambiente.

Para que las empresas de océano rojo puedan sacar ventajas diferenciadoras es necesario que se genere una reciprocidad con el talento humano que muestra compromisos tangibles con la organización, así como con los usuarios o consumidores leales; además de poseer políticas transparentes y compartidas que involucren al medio ambiente.

- Filosofía pensada en la innovación.

Todo gerente cuya empresa se encuentra navegando en un océano rojo debe contar con una direccionalidad estratégica basada en la filosofía empresarial que dio origen al emprendimiento, instrumentando de manera constante la innovación en sus procesos / productos / servicios; con ello, debe buscar darle un sentido de permanencia en el mercado. 
- Diferenciación desde el valor.

El valor del producto o servicio es algo que solo el consumidor reconoce según la utilidad que le dará al mismo. Por ello, las empresas deben reconocer esta diferenciación desde sus procesos internos, y de la cadena de valor que va incorporando en la condición de ser un producto o servicio con características únicas de utilidad para el consumidor.

- Empresas inteligentes.

Las organizaciones inteligentes en el mundo empresarial no son nada novedosa desde el punto de vista de su mención y caracterización; sin embrago, es necesario resaltar la importancia que es el actuar de manera inteligente cuando se trata de empresas que buscan permanecer en el mercado, y más aún cuando estas se encuentran en uno saturado (océano rojo). Las decisiones bien pensadas son señales de una acción inteligente, por lo tanto, la gerencia debe tener siempre presente entre sus decisiones la necesidad de innovar y marcar la diferencia a través de un concepto empresarial único y novedoso.
- Capital intelectual innovador.

El recurso humano intelectual representa un eje estratégico en toda organización empresarial dada su capacidad para pensar, crear e innovar; lo cual resulta en un capital intangible muy valioso al momento de establecer estrategias basadas en la innovación y la aplicación de tecnologías de alto impacto, ambas necesarias para hacer frente a los mercados saturados y las posibilidades de transitar hacia otros océanos con mayor capacidad para crear su propio mercado, con consumidores educados en su propuesta de negocio.

\section{Abriendo espacios para el cierre}

La investigación encuentra un espacio para el cierre en la que muestra sus conclusiones. Es importante destacar que los resultados emergentes brindan nuevos significados en el contexto ecuatoriano para la consolidación de aquellas empresas que navegan en océanos rojos, para lo cual los descriptores gerenciales emergentes dan un sentido axiológico desde las estrategias que se buscan aplicar. 
Como primer aspecto conclusivo, la investigación devela la importancia de incorporar los avances tecnológicos en las empresas de océano rojo dado que sin ella es imposible sobrevivir, más aún cuando las empresas grandes hacen grandes inversiones no solo para mantenerse dominando el mercado, sino que la intención también tiene otro sentido: desequilibrar la competencia.

La segunda vertiente conclusiva revela la necesidad de abrir espacios al capital intelectual como eje conductor para garantizar procesos de desarrollo e innovación (D+i) en empresas que se encuentran en mercados de océano rojo, en las que en algún momento bien pudieran estar en búsqueda de una transición exitosa hacia nuevos mercados.

Por último, es importante considerar que no toda empresa de océano rojo se condiciona a la guerra de precios, también se condiciona a la comodidad del dominio del mercado, en la que se focaliza en solo su permanencia y control sobre otros; esto puede cambiar sí la competencia logra entender la importancia de encontrar espacios de innovación y captación de un recurso humano talentoso que permita transformar la relación de disputar el mercado por precios por un mercado disputado en base a la calidad del producto o servicio.

La investigación deja un espacio para otras iniciativas...hemos concluido.

\section{Bibliografía}

Borghino, M. (2012). El arte de innovar para no morir (El arte de): Cómo sobrevivir en mercados saturados. México: Penguin Random House Grupo Editorial México.

Castro Monge, E. (2010). Las estrategias competitivas y su importancia en la buena gestión de las empresas. Ciencias Económicas, 28(1), $247-276$.

Chan, W. K., \& Mauborgue, R. (2015). La estrategia del océano azul: cómo desarrollar un mercado donde la competencia no tiene ninguna importancia. Bogotá: Profit Editorial.

https://books.google.com.ec/ books?id=b0a5CgAAQBAJ\& printsec $=$ frontcover $\& \mathrm{dq}=\mathrm{OCE}$ ANO+ROJO\&hl=es\&sa $=X \& v$ ed=2ahUKEwilra6C3OzrAhU C11kKHSqPAy8Q6AEwBXoE 
CAQQAg\# $\mathrm{v}=$ onepage $\& \mathrm{q} \& \mathrm{f}=\mathrm{f}$ alse

Chan, W. K., \& Mauborgne, R. (2018). La estrategia del oceáno azul. La transición al océano azul. Más allá de competir. Madrid: Ediciones Urano, S.A.U

Chirinos Cuadros, C. R., \& Rosado Samaniego, J. F. (Enero Diciembre de 2016). Estrategia de diferenciación: el caso de las empresas industriales. Ingeniería Industrial(34), 165 - 174. https://www.redalyc.org/pdf/3 374/337450992008.pdf

Gadamer, $\mathrm{H}$.

G. (1998). Fundamentos de la Hermenéutica. Siglo $\mathrm{XX}$ Editores.

Gómez, D. (2017). Bueno, Bonito y Carito: cómo diferenciarse para dejar de competir por precio. Bogotá - Colombia: Penguin Random House Grupo Editorial.

Hernández Escobar, A. A., Ramos Rodríguez, M. P., Placencia López, B. M., Indacochea Ganchozo, B., Quimis Gómez, A. J., \& Moreno Ponce, L. A. (2018). Metodología de la Investigación Científica. Editorial Área de Investigación y Dessarrollo, S.L.

https://books.google.com.ec/ books?id=y3NKDwAAQBAJ\& printsec=frontcover \&hl=es\&s ource=gbs_ge_summary_r\&c $\mathrm{ad}=0 \# \mathrm{v}=$ onepage $\& \mathrm{q} \& \mathrm{f}=\mathrm{false}$

Muñoz Rocha, C. (18 de Octubre de 2015). Metodología de la investigación. México D,F: Editorial Progreso S.A. https://books.google.com.ec/ books?id=DflcDwAAQBAJ\&p rintse $\mathrm{c}=$ frontcover $\& \mathrm{dq}=\mathrm{mu} \%$ C3\%B1oz+rocha $+2015 \& \mathrm{hl}=\mathrm{e}$ s\&sa $=X \& v e d=2 a h U K E w j 6 r e$ F0PvrAhWIrFkKHagDAbsQ6 AEwAHoECAAQAg\#v=onepa ge\& $q=$ mu\%C3\%B1 oz\%20roc ha\%202015\&f=false

Oppenheimer, A. (2014). ¡Crear o morir! La esperanza de Latinoamérica y las cinco claves de la innovación. México: Penguin Random House Grupo Editorial México.

Ortiz, D. (2020). Innovación estratégica para empresas de océano rojo. Alternativas para una transición exitosa. Entrevista en profundidad.

Porter, M. E. (2015). Ventaja Competitiva: Creación y sostenimiento de un desempeño superior. México: Grupo Editorial Patria, S.A De C.V. https://books.google.com.ec/ books?id=wV4JDAAAQBAJ\& printsec $=$ frontcover $\& d q=$ vent aja+competitiva+de+michael +porter\&hl=es\&sa=X\&ved $=2 a$ hUKEwiTzIGznK_sAhWHjVk KHfvXAOcQ6AEwAHoECAU 
QAg\#v=onepage $\& q=$ entaja \%20competitiva\%20de\%20mi chael\%20porter \&f=false

Pinto, V.H. (2020). Innovación estratégica para empresas de océano rojo. Alternativas para una transición exitosa. Entrevista en profundidad.

Pulgarín, S., \& Pineda, L. (2011). La innovación estratégica: su caracterización y un posible enfoque desde las ciencias de la complejidad. Criterio Libre, 9(15), 173 - 192. https://revistas.unilibre.edu.co /index.php/criteriolibre/article/ view/1207/936

Sheetz Runkle, B. (2019). El arte de la guerra. La pequeña y mediana empresa. Derrote a la competencia y domine el mercado con las estrategias magistrales de Sun Tzu. Estados Unidos de América: Grupo Nelson.

Vegas M., H. (2016). La teoría fundamentada como herramienta metodológica para el estudio de la gestión pública local. Revista Venezolana de Gerencia., $21(75)$.

https://produccioncientificaluz .org/index.php/rvg/article/view /21891/21636 